\title{
ORIGINE BOTANIQUE ET CARACTÉRISTIQUES PHYSICOCHIMIQUES DES MIELS
}

\author{
E. C. BARBIER et Claude-Yolande PANGAUD. \\ Laboratoire apicole, Nice.
}

\section{SOMMAIRE}

Les miels, ayant une origine botanique bien définie, possèdent des caractéristiques particulières de couleur, de pH initial, et de courbe de neutralisation des acides libres.

Ces caractéristiques apportent un complément nécessaire ì l'analyse pollinique pour la recherche de l'origine botanique d'un miel.

\section{IN'TRODUC'TION.}

Les apiculteurs ont depuis longtemps attribué aux divers miels récoltés dans leurs ruches et considérés comme provenant d'une plante mellifère bien définie, certains caractères parmi lesquels la couleur et le mode de cristallisation ont le plus attiré leur attention.

I e but de ce travail a été de rechercher dans quelle mesure 1'origine botanique d'un miel pouvait influer sur les caractéristiques suivantes : couleur, $\mathrm{pH}$ initial et neutralisation des acides libres.

D'une façon générale les observations faites sur la couleur par les praticiens sont valables. L'examen des exceptions qui ont pu être relevées, a montré que l'origine botanique attribuée aux échantillons était erronée, ou que, le plus souvent, les miels en question provenaient de mélanges naturels réalisés par les abeilles au cours de leurs récoltes successives.

Les sources de matières sucrées sont si nombreuses, et leur production est d'une telle variabilité (qu'il n'est pas étonnant que les miels soient très différents les uns des autres et qu'il soit si difficile de déterminer leur origine botanique autrement que par une étude rigoureuse.

\section{I. -- ORIGINI: BO'TANIQUE: DES MIELS}

1)ans l'état actuel de nos connaissances, l'analyse pollinique apparait comme la seule méthode qui puisse apporter des éléments permettant de juger de cette origine.

Pour déterminer l'origine botani(jue des 860 échantillons de miel qui nous ont 
été adressés par les apiculteurs ( $\left.{ }^{1}\right)$ la méthode d'analyse pollinique décrite par MAURIzıo (I949) a été utilisée en y apportant toutefois quelques modifications de détail :

a) Les préparations microscopiques sont faites en utilisant $20 \mathrm{~g}$ de miel ou $40 \mathrm{~g}$ pour les échantillons pauvres en pollen $\left(_{2}\right)$.

b) Dans le but d'obtenir des préparations plus claires, une première centrifugation est faite avec de l'eau acidulée $\left({ }^{3}\right)$. L'emploi d'une solution à $5 \mathrm{ml}$ d'acidesulfurique par, litre suivi d'une centrifugation à l'eau distillée nous a donné de bons résultats sans nuire à la coloration à la fuchsine.

c) Les tubes utilisés pour la centrifugation sont en matière plastique et démontables; ils seront décrits ultérieurement.

\section{Pureté des échantillons.}

Pour les miels d'Allemagne ZANDER considérait qu'un miel était " unifloral " c'est-à-dire récolté principalement sur une espèce végétale, lorsque dans son spectre pollinique les pollens de la plante principale atteignaient au moins 45 p. Ioo.

Toutefois MaURIzIo (I939 - I949 - I955 - I958) constatait que ce taux devait être modifié en fonction de 1'origine botanique des miels : élevé à plus de 70 p. Ioo pour les miels de Castanea ou de Myosotis, ou abaissé à moins de 40 p. Ioo pour ceux de Robinia, à 29 p. Ioo pour ceux de Rosmarinus et même à 7 p. Ioo pour ceux de Lavandin.

Ces différences considérables trouvent leur explication dans les différences anatomiques des fleurs (BARBIER I954) et dans le rapport des productions nectarifère et pollinifère. De plus le butinage des pollens destinés à l'alimentation du couvain (Louveaux I958), 1'utilisation des pollens en réserve dans la ruche, ainsi que les opérations d'extraction, contribuent à rendre délicate l'interprétation des résultats de l'analyse pollinique.

Dans ces conditions nous avons choisi, pour chaque origine botanique, les échantillons présentant le taux le plus élevé possible pour le pollen principal, ceci a fin d'éviter toute erreur d'interprétation.

Dans le présent travail ces miels seront appelés " miels types "; c'est sur eux que seront évaluées les caractéristiques suivantes : couleur, $\mathrm{pH}$ initial et pente de la tangente à la courbe de neutralisation.

Mais nous avons pu remarquer que des miels n'ayant que $30 \mathrm{p}$. Ioo de pollen de Robinia, de 25 à 9 p. Ioo de Rosmarinus et même 4 p. Ioo de Lavandin se sont comportés comme les échantillons de même origine qui présentaient une proportion beaucoup plus grande en ces pollens principaux. Cette particularité étant due sans aucun doute à la présence accidentelle de pollens secondaires non accompagnés des miels correspondants.

(1) Nous remercions ici les nombreux apiculteurs qui nous ont adressé depuis plusicurs annés les échantillons de leurs récoltes.

(2) Nous remercions également Melle MaL aussene qui a réalisé les préparations microscopiques d'un certain nombre d'échantillons.

( $\left.{ }^{3}\right)$ Procédé préconisé par Mr. Louveaux (communication verbale). 


\section{II. - ÉVALUATION DES CARAC'TÉRISTIQUES.}

\section{Couleur}

Après liquéfaction des échantillons, leur couleur est déterminée par comparaison avec une série d'étalons, constitués par des solutions d'iode à concentration croissante (BARBIER E. C. et VALIN J. I957).

La sensibilité de l'œil étant variable selon le logarithme de l'intensité de la couleur, il en résulte que I I, $9 \mathrm{mg}$ d'iode par litre séparent Blanc I et Blanc II, alors qu'il en faut $9900 \mathrm{mg}$ entre Foncé II et Foncé III.

(On trouvera au tableau I un classement de quelques miels " types " d'après leur couleur).

TABLEAU I

Couleur de quelques miels " types"

\begin{tabular}{|c|c|c|c|c|}
\hline \multicolumn{3}{|c|}{ Couleur } & \multicolumn{2}{|r|}{ Miel } \\
\hline $\begin{array}{l}\text { Appellation } \\
\text { commerciale }\end{array}$ & Mg. iode par litre & $\begin{array}{c}\text { Échelle } \\
\text { logarithmique }\end{array}$ & $\begin{array}{c}\text { Échantillon } \\
\text { No }\end{array}$ & Origine Botonique \\
\hline $\begin{array}{cc}\text { Blanc } & \text { I....... } \\
& \\
- & \text { II } \ldots \ldots . \\
- & \text { III........ }\end{array}$ & $\begin{array}{l}19,5 \text { à } 31,4 \\
31,4 \text { à } 50,7 \\
50,7 \text { à } 81,5\end{array}$ & $\begin{array}{l}13 \text { à } 15 \\
15 \text { à } 17 \\
17 \text { à } 19\end{array}$ & $\begin{array}{l}256 \\
818 \\
440 \\
763\end{array}$ & $\begin{array}{l}\text { Rosmarinus officinalis L. } \\
\text { Robinia Pseudacacia L. } \\
\text { Hedysarum coronarium L. } \\
\text { Lavandin }\end{array}$ \\
\hline $\begin{array}{cc}\text { Jaune } & \text { I. . . . . . } \\
- & \text { II . . . . } \\
- & 11 \mathrm{I} \ldots\end{array}$ & $\begin{array}{l}x 1,5 \text { à } 131,6 \\
131,6 \text { à } 212 \\
212 \text { à } 342\end{array}$ & $\begin{array}{l}19 \text { à } 21 \\
21 \text { à } 23 \\
23 \text { à } 25\end{array}$ & $\begin{array}{l}345 \\
36 t \\
100\end{array}$ & $\begin{array}{l}\text { Onobrychis viciifolia Scop. } \\
\text { Satureia montana .L. } \\
\text { Lavandula officinalis Chaix }\end{array}$ \\
\hline $\begin{array}{cc}\text { Doré } & \text { I } \ldots \ldots \ldots \\
- & \text { II } \ldots \ldots \ldots \\
- & \text { III...... }\end{array}$ & 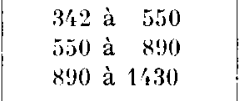 & $\begin{array}{l}25 \text { à } 27 \\
27 \text { à } 29 \\
29 \text { à } 31\end{array}$ & $\begin{array}{l}594 \\
843\end{array}$ & $\begin{array}{l}\text { Calluna mulgaris L. } \\
\text { Erica arborea } \mathrm{L} .\end{array}$ \\
\hline $\begin{array}{cr}\text { Ambre } & \text { I } \ldots \ldots \ldots \\
- & \text { II } \ldots \ldots . . \\
- & \text { III } \ldots \ldots .\end{array}$ & $\begin{array}{l}1430 \text { à } 2310 \\
2310 \text { à } 3720 \\
3720 \text { à } 6000\end{array}$ & $\begin{array}{l}31 \text { d̀ } 34 \\
34 \text { à } 36 \\
36 \text { à } 38\end{array}$ & 278 & (Manne) Abies pectinata D.C. \\
\hline $\begin{array}{cr}\text { Foncé } & \text { I } \ldots \ldots . \\
- & \text { II } \ldots \ldots . \\
- & \text { III } \ldots \ldots \ldots\end{array}$ & $\begin{array}{r}6000 \text { à } \quad 9650 \\
9650 \text { à } 15500 \\
15500 \text { à } 25400\end{array}$ & $\begin{array}{l}38 \text { à } 40 \\
40 \text { à } 42 \\
42 \text { à } 44\end{array}$ & 39 & Miel de Guinée \\
\hline
\end{tabular}

Cette particularité de la vision humaine a pour conséquence que la détermination de la couleur chez les miels clairs est très instructive. Elle permet de déceler dans ceux-ci des traces de miel foncé, traces que l'analyse pollinique seule, compte tenu des remarques précédentes, n'aurait pu mettre en évidence, alors que chez les miels foncés elle est secondaire car la quantité de miel clair susceptible d'apporter une modification à la couleur, est si importante que l'analyse pollinique permettra de la déceler facilement.

D'une façon théorique on peut calculer la modification de couleur, apportée à 
un miel clair par la présence d'un miel foncé. Les résultats donnés au tableau $n^{0} 2$, ont été confirmés expérimentalement; ils ont été obtenus en mélangeant à 90 parties de miel de Lavandin très pur (couleur Blanc III $=63,5 \mathrm{mg}$ d'iode par litre), à Io parties d'un miel récolté dans 1'Esterel, principalement sur Erica arborea $\mathrm{I}_{\text {. }}$ et dont la couleur, classée Doré III, était égale à celle d'une solution titrant I I33 mg d'iode par litre.

TABIEAU 2

Modification de la couleur, d'un miel de lavandin, due à la prósence d'un miel fonct.

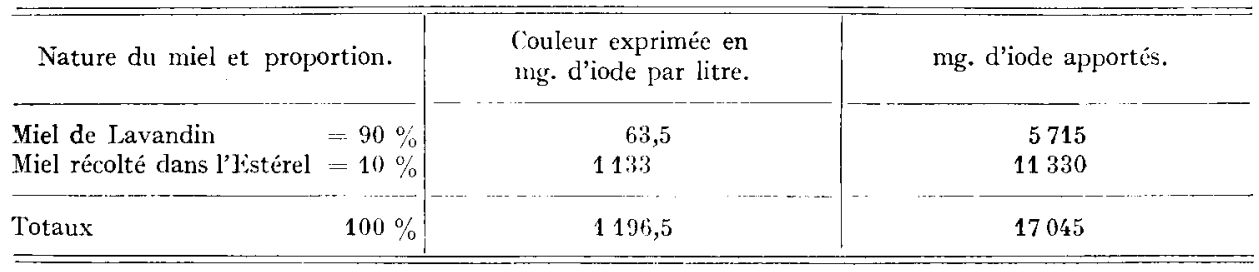

La couleur du mélange sera égale à celle d'une solution titrant $x$ 70,45 mg d'iode par litre ce qui correspond à Jaune II.

Le même calcul permet encore de se rendre compte que la présence de ro p. Ioo de miel clair dans un miel foncé serait sans grande influence sur la couleur finale. Dans le cas présent le titre d'iode correspondant passerait de I I $33 \mathrm{mg}$ à I $026 \mathrm{mg}$.

Il convient encore de signaler que la couleur d'un miel n'est pas fixe et qu'elle varie dans le temps comme l'a montré MrLUm (I949). Selon l'origine botanique cette variation sera plus ou moins rapide; ainsi un miel récolté principalement sur Erica arborea $\mathrm{L}$. depuis quelques années, avait au moment de sa mise en tube une couleur égale à celle d'une solution titrant 3 I $80 \mathrm{mg}$ d'iode par litre, quatre ans après sa couleur correspondait à $25400 \mathrm{mg}$ d'iode ; par contre d'autres échantillons conservés de la même façon et récoltés notamment sur Rosmarinus officinalis I. n'avaient pas hangé de couleur d'une façon sensible durant cette même période.

TABLEAU 3

Miels "types "présentant un pH initial de valeur anormale.

\begin{tabular}{|c|c|c|}
\hline Plante mellifère & $N^{T} \mathrm{de}$ l'échantillon & plI \\
\hline 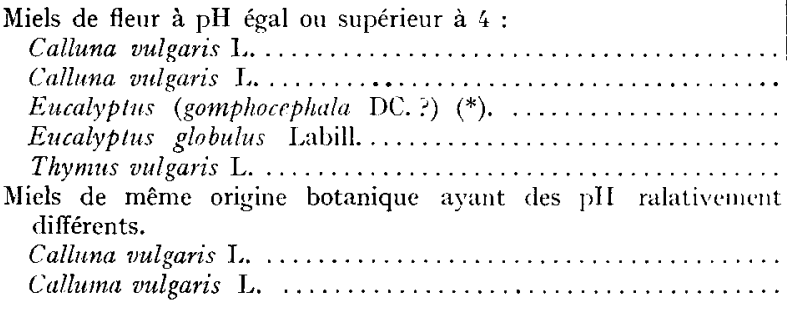 & $\begin{array}{l}596 \\
691 \\
743 \\
857 \\
7 \div 7\end{array}$ & $\begin{array}{l}5,2 \\
4,7 \\
4,0 \\
1,2 \\
1,0 \\
1,2 \\
5,2 \\
4,0\end{array}$ \\
\hline
\end{tabular}

$\left(^{*}\right)$ Le pollen de cet Eucalyptus n'existant pas dans notre collection nous ne pouvons confirmer l'origine de cet échantillon qui nous à été arlressé par Mr. II.wcourt (Maroc). 


\section{pH initial du miel en solution.}

L,e $\mathrm{pH}$ initial du miel est mesuré sur une solution à r/Io (poids/volume). L'appareil utilisé à cet effet est un $\mathrm{pH}$ mètre Carpeni et Constant étalonné sur une solution tampon de Michaëlis à $\mathrm{pH}=4,62$ et sur l'étalon de la Maison Jouan à $\mathrm{pH}=3,57$.

La mesure de ce $\mathrm{pH}$ initial ( $\mathrm{pH}$ i sur la figure $\mathrm{I}$ ) est faite aussitôt après la dissolution du miel. D'une façon générale ce $\mathrm{pH}$ varie de 3,3 à 4,0 pour les miels de fleurs et de 4,5 à 5,5 pour les miellats, et reste à peu près constant pour les miels de même type, comme 1'avait noté CHISTOV (I954).

Toutefois ces indications présentent des exceptions dont on trouvera quelques exemples au tableau 3, où sont donnés les pH de miels considérés comme "types", tout au moins dans la limite de nos connaissances actuelles.

\section{Courbe de neutralisation}

La technique d'obtention d'une telle courbe est la suivante : (PANGaud ig6o).

- Peser $5 \mathrm{~g}$ de miel, étendre à $50 \mathrm{ml}$ avec de l'eau distillée (solution à $\mathrm{I} / \mathrm{I}$, poids/volume).

- Prendre $25 \mathrm{ml}$ de cette solution.

- Pendant toute la suite des opérations, brasser la solution à l'aide d'un agitateur magnétique.

- lire le $\mathrm{pH}$ initial ou $\mathrm{pH}$ i.

- Verser la solution de soude $\mathrm{Na} \mathrm{OH} \mathrm{N} / 20$ à la burette de précision en quantité variable selon la marche de la neutralisation de 0,02 à $0,2 \mathrm{ml}$ à la fois et mesurer aussitôt le pH.

Le pH mètre doit être étalonné avant et après chaque série de dosages et la température comprise entre $\mathrm{I}^{\circ}-20^{\circ} \mathrm{C}$. La courbe de neutralisation est alors construite en portant en abscisse les millilitres de solution de soude versés et en ordonnée les $\mathrm{pH}$ correspondants (Voir figure I). La pente de la tangente $\mathrm{N}$., tracée au point équivalent $\mathrm{E}$. de la courbe de neutralisation, donne une notion de la force des acides. Plus cette pente sera basse plus les acides seront faibles et inversement.

La valeur de cette pente peut être représentée par l'augmentation de $\mathrm{pH}$ que provoquerait sur la tangente $\mathrm{N}$ une addition de $0,2 \mathrm{ml}$ de $\mathrm{Na} \mathrm{OH} \mathrm{N} / 20$. Dans les conditions de dosages et avec les électrodes utilisées (électrode de verre type EVB et électrode de référence type ERB) entre $\mathrm{pH}=2$ avec addition d'acide chlorhydrique et $\mathrm{pH}=$ Io, avec addition de soude, la courbe n'a jamais présenté de paliers intermédiaires correspondant à la neutralisation distincte d'acides différents. Cela tient vraisemblablement à ce que les acides présents dans les miels sont de force voisine.

Les dosages effectués sur des échantillons appartenant à divers types de miel montrent de grandes différences dans la valeur de la pente de neutralisation. C'est ainsi que cette valeur passe de 3,7 pour un miel de Romarin ( $\mathrm{n}^{\circ} 256$ ) à 0,5 pour une manne de Mélèze ( $\left.\mathrm{n}^{\circ} 474\right)$. Une pente de 0,2 a même été obtenue avec un miellat non identifié récolté dans les Pyrénées Orientales. Ces différences indiquent que les acides de ces miels ne sont pas identiques ou que les proportions relatives des divers acides sont différentes. 


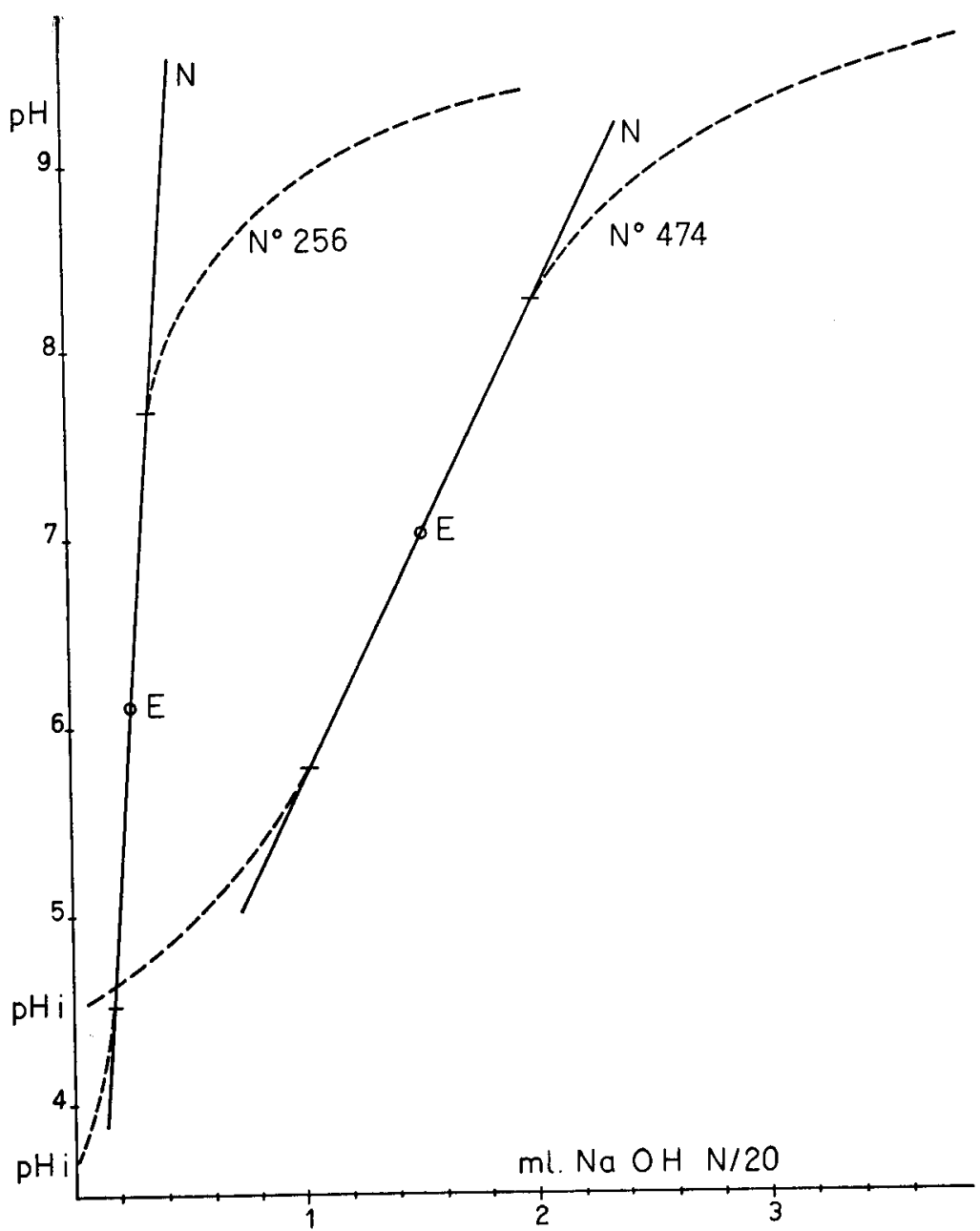

FIG. I.

TABIEAU 4

Valeur de la pente pour des miels types de même nature et d'origine géographique différente.

\begin{tabular}{|c|c|c|c|}
\hline Nature & $\mathrm{N}^{0}$ & Origine & Pente \\
\hline Robinier Robinia Pseudacacia I....... & $\begin{array}{l}818 \\
820\end{array}$ & $\begin{array}{l}\text { La Ferté-sous-Jouarre (S. \&. M.) } \\
\text { La Chapelotte-de-Villeneuve la Guyard } \\
\text { (Yonne) }\end{array}$ & $\begin{array}{l}2,5 \\
2,5\end{array}$ \\
\hline 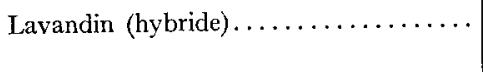 & $\begin{array}{l}389 \\
794\end{array}$ & $\begin{array}{l}\text { Valensole (B. A.) } \\
\text { Valréas (Vaucluse) }\end{array}$ & $\begin{array}{l}1,9 \\
2,0\end{array}$ \\
\hline Callune Calluna vulgaris L.......... & $\begin{array}{l}596 \\
614\end{array}$ & $\begin{array}{l}\text { Lac Servière (Puy de Dôme) } \\
\text { Lacanau (Gironde) }\end{array}$ & $\begin{array}{l}1,2 \\
1,1\end{array}$ \\
\hline
\end{tabular}


Des nombreux dosages effectués, il ressort que la valeur de cette pente semble être constante ou qu'elle ne varie que dans des limites étroites pour des miels de même origine botanique même si leur origine géographique est différente; on en trouvera quelques exemples au tableau 4.

\section{Origines des acides des miels} miels.

Deux origines ont été avancées pour expliquer la présence des acides dans les

a) Origine botanique. BEUTLER (I953) a signalé que les nectars sont acides et que suivant la plante productrice ils possèdent une acidité différente, certains même pouvant être basiques.

b) Origine enzymatique. COCKER (I95I) a montré que les abeilles ont la possibilité de provoquer dans les miels la formation d'acides. En donnant en nourrissement un sirop de sucre à $\mathrm{pH}=7,4$ il a constaté que les réserves constituées par les abeilles avaient un $\mathrm{pH}=4,3$.

Io $p H$ de divers nectars.

A l'aide d'un papier indicateur universel de $\mathrm{pH}$, nous avons pu relever, avec une certaine approximation, le $\mathrm{pH}$ de divers nectars prélevés dans les fleurs de plantes cultivées en serre $\left({ }^{1}\right)$ ou en plein air.

Les observations portées au tableau 5 confirment que les nectars sont acides et que leur acidité est variable selon leur origine. Mais nous n'avons pas trouvé de nectar à réaction basique; le $\mathrm{pH}$ se tient en général aux environs de 4,5 et chez les fleurs âgées, où le nectar a séjourné plusieurs jours, le $\mathrm{pH}$ tend à s'abaisser.

TABLEAU 5

pH de divers nectars (au papier universel).

\begin{tabular}{|c|c|c|c|}
\hline Plante & Age des fleurs & Lisu de culture & pH approximatif \\
\hline Lavandin (hybride). & jeune & Jin serre & 4,5 \\
\hline Lavandin (hybride). & âgés & -- & 3,5 \\
\hline Strelitzia Regmae Banks. & jeune & - & 4,5 \\
\hline Primula Kewensis........ & - & - & 6 \\
\hline Rosmarinus offinalis L.... & - & en plein air & 4 \\
\hline Eucalypius globulus Labill. ................ & - & - & 4,5 \\
\hline Datura sanguinea Ruiz et Pav... & - & - & 5,5 \\
\hline Citrus aurantium L. $\ldots \ldots \ldots \ldots \ldots$. & - & - & 4,5 \\
\hline
\end{tabular}

\section{$2^{\circ}$ Comparaison entre un nectar et un miel.}

Par ailleurs nous avons pu comparer au point de vue de leur $\mathrm{pH}$ et de leur courbe de neutralisation un nectar et un miel d'Eucalyptus globulus Labill.

a) Prélèvement du nectar. - Un certain nombre de rameaux à fleurs ont été coupés sur les arbres vers $9 \mathrm{~h}$ le 4 février rg6o et transportés au Laboratoire; le nectar a été prélevé à la pipette, putis conservé à la température de $2^{\circ} \mathrm{C}$.

(1) Nous remercions ici Monsieur le Directeur des Serres de la Ville de Nice qui nous a permis de faire les prélèvements nécessaires. 
Les rameaux ont été ensuite placés, l'extrémité dans l'eau, sous une hotte à Io $-20^{\circ}$ et à humidité relative élevée (90 à $95 \mathrm{p}$. Ioo) de façon à favoriser la sécrétion nectarifère. Chaque matin le nectar était prélevé et conservé à basse température.

Les divers nectars ainsi que leur mélange, correspondaient aux données figurées au tableau 6 .

\section{TABLEAU 6}

Composition et $p H$ des nectars d'Eucalyptus globulus Labill.

\begin{tabular}{|c|c|c|c|c|}
\hline \multirow{2}{*}{ Date du prélèvement } & \multirow{2}{*}{ Nectar en mg. } & \multicolumn{2}{|c|}{ Matière sèche } & \multirow{2}{*}{$\mathrm{pH}$} \\
\hline & & en $\%$ & en $\mathrm{mg}$. & \\
\hline $\begin{array}{l}4 / 2 / 609 h \ldots \ldots \ldots \ldots \\
5 / 2 / 609 \mathrm{~h} \ldots \ldots \ldots \ldots \\
6 / 2 / 609 \mathrm{~h} \ldots \ldots \ldots \ldots\end{array}$ & $\begin{array}{r}965 \\
10.729 \\
7.450\end{array}$ & $\begin{array}{r}14,7 \\
10,0 \\
7.0\end{array}$ & $\begin{array}{r}141,8 \\
1.072,9 \\
521,5\end{array}$ & $\begin{array}{c}\text { (au papier universel) } \\
\text { environ } 4,5 \\
\text { environ } 5,5 \\
\text { environ } 4,5\end{array}$ \\
\hline Mélange $\left(^{*}\right) \ldots \ldots \ldots$ & 19,137 & 9 & $1.732,3$ & (au $\left.{ }_{1}\right) \mathrm{H}$ mètre) 4,3 \\
\hline
\end{tabular}

$\left(^{*}\right)$ En tenant compte des pertes.

b) Obtention du miel. - A l'île Sainte-Marguerite près de Cannes (A-M.), les Eucalyptus globulus Labill. sont nombreux et au moment où ils fleurissent il ne peut y avoir d'autre miellée. Dans une ruche installée dans cette île, un cadre vide a été introduit le I3 février Ig6o et retiré Io jours après.

Dans ce cadre les abeilles avaient emmagasiné environ $400 \mathrm{~g}$ de miel non encore operculé qui répondait aux caractéristiques suivantes :

Échantillon $\mathrm{n}^{\circ} 857$.

Matière sèche $=72,6 \mathrm{p}$. Ioo.

Couleur $=$ égale à celle d'une solution d'iode à $\mathrm{N} / 600$ et titrant $2 \mathrm{I} 2 \mathrm{mg}$ d'iode par litre (ou N/530 $=240 \mathrm{mg}$ pour le miel ramené à $82 \mathrm{p}$. Ioo de matiète sèche).

Richesse pollinique $=58600$ grains de pollen pour Io $g$ de miel (ou 67 ooo pour le même miel à $82 \mathrm{p}$. Ioo de matiète sèche).

Spectre pollinique $=$ Eucalyptus globulus Labill Ioo p. Ioo avec présence, hors comptage, de très rares pollens d'Erica arborea L et d'Acacia dealbata. Link.

Afin de permettre la comparaison des caractéristiques de ces deux échantillons les valeurs portées au tableau 7 ont été ramenées par le calcul à celles d'un miel à 82 p. Ioo de matière sèche.

\section{TABLEAU 7}

Caractéristiques d'un nectar et d'un miel

d'Eucalypius globulus Labill. (Ramenés à 82 p. 100 de M.S.)

\begin{tabular}{|c|c|c|c|c|}
\hline Nature de l'échantillon & $\mathrm{pH}$ initial & Milli équivalent \% & $\begin{array}{l}\mathrm{pH} \text { du point } \\
\text { équivalent }\end{array}$ & Pente \\
\hline $\begin{array}{l}\text { Nectar } \ldots \ldots \ldots \ldots \ldots \ldots \\
\text { Miel } n^{\circ} 857 \ldots \ldots \ldots \ldots \ldots\end{array}$ & $\begin{array}{l}4,3 \\
4,2\end{array}$ & $\begin{array}{l}1,5 \\
1,9\end{array}$ & $\begin{array}{l}6,{ }^{\prime} k \\
6,6\end{array}$ & $\begin{array}{l}1,1 \\
1,0\end{array}$ \\
\hline
\end{tabular}


L'examen de ces résultats permet de constater que l'acidité du miel est plus forte que celle du nectar ( $I, 9$ milli-équivalent contre $I, 5)$ sans que les autres caractéristiques et notamment la pente soient sensiblement modifiées.

En conséquence il ressort de la lecture des tableaux 4 et 7 que dans les conditions normales de la récolte, la pente de la tangente à la courbe de neutralisation peut être considérée comme une caractéristique de 1'origine botanique d'un miel.

\section{III. - RÉSULTATS ET DISCUSSIONS.}

Sur les échantillons dont nous disposions nous n'avons utilisé que ceux possédant un spectre pollinique nettement marqué. On constate aussitôt qu'à une origine botanique donnée correspond une gamme de couleur plus ou moins étendue, mais avec convergence vers un couleur particulière pouvant être considérée comme caractéristique de l'origine botanique.

En effet, si comparativement à la couleur on examine les résultats de l'analyse pollinique des échantillons, on peut remarquer, pour chaque origine botanique, que les miels présentant une couleur égale ou très voisine de la couleur type ont également une composition pollinique très voisine, caractérisée par un taux élevé de pollens de la plante principale.

Par contre tous les miels qui s'éloignent de cette couleur type ont un spectre pollinique plus ou moins altéré par une proportion élevée de pollens secondaires ou par la présence d'algues ou de fructifications de champignons, indiquant par cela mêtme que ces échantillons ne sont pas purs mais qu'ils sont le résultat de mélanges avec d'autres miels ou avec des miellats.

La même constatation peut être faite avec la courbe de neutralisation.

\section{A. - Miels de lavandin.}

Nous examinons particulièrement le cas des miels de Lavandin qui présentent un intérêt certain pour notre région du Sud-Esst. Grâce à leur couleur claire et à leur mode d'obtention basé principalement sur la transhumance, ces miels constituent un matériel de choix pour l'étude présente ; en effet, les étapes de butinage dans des zones à végétation bien différenciée sont connues et les miels qui peuvent y être récoltés perturbent fortement le spectre et la richesse pollinique par leurs pollens qui sont en général facilement identifiables.

Sur les 860 échantillons reçus 76 nous ont été adressés sous la dénomination de miel de Lavandin. Parmi ceux-ci 45 pouvaient être considérés par l'analyse pollinique comme étant des miels à forte dominance de Lavandin ; mais la détermination de la couleur en éliminait 20 , qui présentaient une teinte plus ou moins éloignée de la couleur type. Sur les 25 restants, I5 étaient inutilisables pour l'établissement de la courbe de neutralisation soit à cause de leur mauvaise conservation dûe à une humidité trop élevée, soit à cause de leur utilisation à d'autres fins. Aussi cette courbe ne fut-elle tracée que pour Io miels sélectionnés qui donnèrent une pente de valeur très homogène $I, 9$ à 2,0 (un seul échantillon à $I, 8$ ).

La courbe de neutralisation fut également tracée pour I5 miels ayant seulement une dominance Lavandin ; leur valeur de pente présente une grande dispersion 
allant de I,7 à I,3. L'analyse pollinique de ces miels montrait d'ailleurs d'une façon bien nette la présence de pollens provenant des étapes antérieures de transhumance; leur présence liée à une couleur plus foncée et à la modification de la pente était la preuve que ces pollens étaient accompagnés des miels correspondants, comme le montre le tableau 8 dans lequel :

a) L'échantillon $n^{\circ} 472$ correspond à un miel extrait à la pipette dans un cadre rempli pendant la miellée du Lavandin.

On notera dans cette analyse la pauvreté en pollens totaux et 1'importance du Lavandin qui sont dûes au mode d'obtention de l'échantillon. Parmi les pollens d'ac-

\section{TABLEAU 8}

Examen comparé de 3 miels de Lavandin.

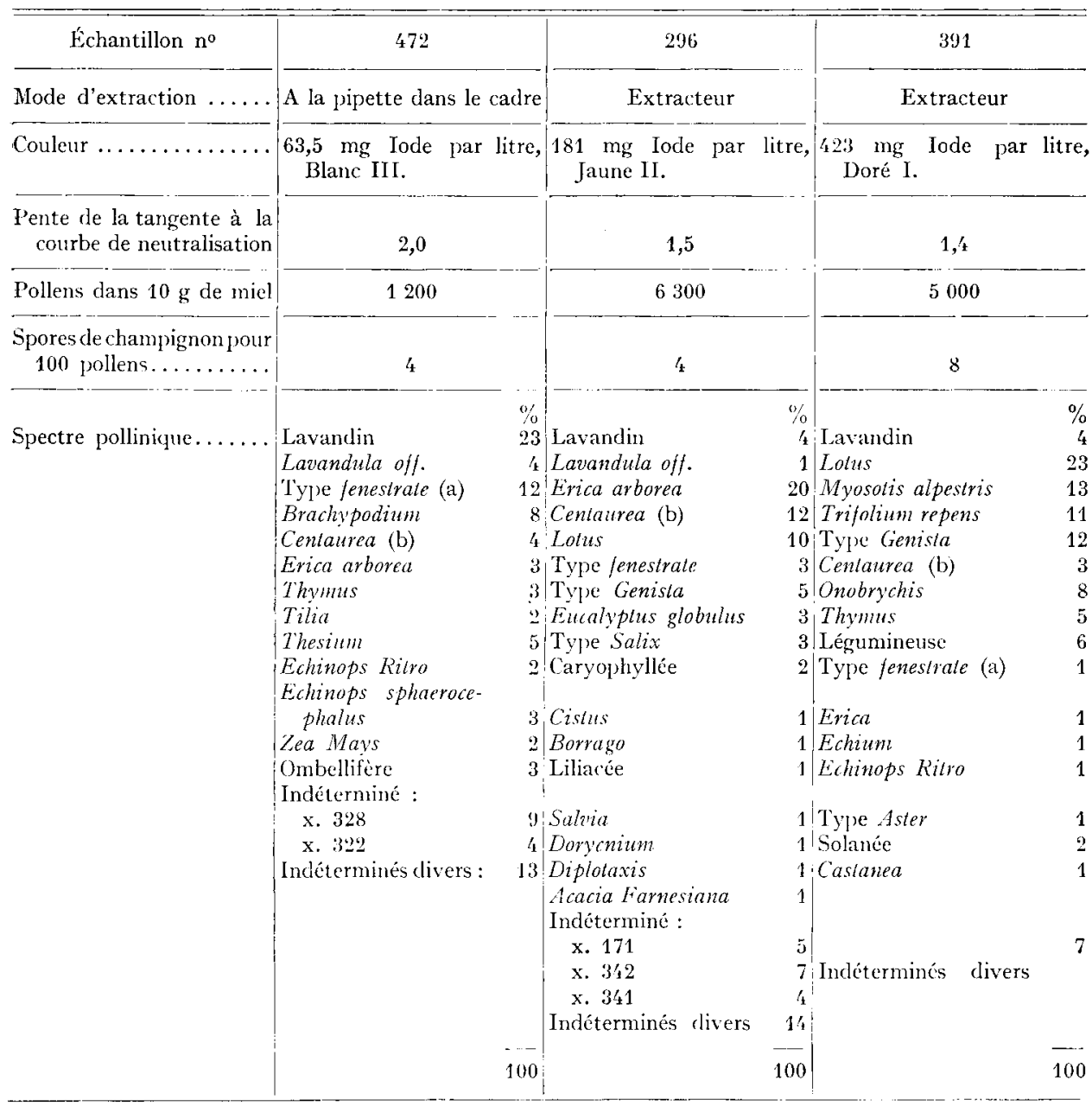

(a) Type fenestrate désigne un pollen correspondant à la définition clonnée par FAkgri et IvkRsEN (1950).

(b) Centurea du type C. aspera L. 
compagnement importants, on trouve ici Brachypodium, Thesium, deux indéterminés et une forme "fenestrate"; on trouve aussi parfois Myrtus. Chez certains échantillons ces pollens atteignent accidentellement des taux élevés à cause de l'abondance de ces plantes dans les maquis voisins ou dans les terrains revenus à la friche ou mal cultivés. Avec Centaurea du type $C$. aspera L. et Echinops ces pollens constituent des éléments précieux pour l'établissement de l'origine géographique.

La présence d'Erica arborea L. s'explique par le fait que la ruche avait hiverné dans le massif des Maures où cette plante est abondante. L'utilisation des réserves pour l'alimentation du couvain, a provoqué l'apparition de ce pollen dans le spectre pollinique, mais en très faible proportion.

b) L'échantillon $n^{\circ} 296$ est un miel d'extracteur, il provient de ruches ayant hiverné sur le littoral varois, et dont la récolte a été faite après la miellée du Lavandin.

Sa composition pollinique montre bien cette étape de transhumance par la présence de : Erica arborea I., Eucalyptus globulus Labill., Acacia Farnesiana Link.; et ses caractéristiques confirment la présence d'une certaine proportion de miel récolté à cette étape de transhumance $\left({ }^{1}\right)$.

c) L'échantillon $n^{0} 39 \mathrm{r}$ est également un miel d'extracteur récolté dans les mêmes conditions que le $\mathrm{n}^{0} 296$ mais il provient de ruches ayant transhumé dans les Alpes. Cette étape est caractérisée par Trifolium repens L., Myosotis alpestris I., Lotus, Onobrychis.

On trouvera en outre au tableau 9 la composition pollinique et les caractéristiques de deux miels de Lavandin très purs d'extracteur. Leur richesse pollinique est plus élevée que celle de l'échantillon $n^{\circ} 472$, leur spectre pollinique est différent mais leur couleur et leur pente sont inchangées; ce qui indique que les pollens en surnombre dans ces deux miels sont là accidentellement et qu'ils ne sont pas accompagnés des miels correspondants, cet enrichissement en pollen étant probablement dû aux opérations d'extraction.

\section{B. - Miels divers.}

Pour certains miels nous disposions d'un nombre suffisant d'échantillons unifloraux (Romarin 2 - Sarriette 2 - Callune 6 - Robinier 3 - Bruyère blanche 2 Sainfoin 2) et d'échantillons moins purs, pour pouvoir considérer les premiers comme miels "types" et en dégager les caractéristiques par analogie avec ce qui avait été constaté sur les miels de Lavandin, c'est-à-dire mêmes modifications de la couleur et de la pente.

Pour d'autres, bien que ne pouvant suivre d'une façon aussi nette sur plusieurs échantillons l'influence des mélanges, leurs caractéristiques ont été considérées comme valables grâce au taux élevé du pollen principal dans le spectre des échantillons suivants. Hedysarum $2-$ Eucalyptus globulus Labill. $2-$ E. (gomphocephala?) I Colza I - Thym 2.

Pour les mannes n'ont été publiés que les résultats des échantillons récoltés personnellement ou ayant une origine certaine et présentés en section.

Enfin pour les miellats n'ont été retenus que les résultats obtenus avec des échan-

(1) Ce miel ne peut être extrait complètement des ruches avant le transport car il se trouve dans les cadres à couvain et autour de celui-ci. Après la miellée sur lavandin alors que le couvain a disjaru, seul le tri rles cadres permettrait son ćlimination. 


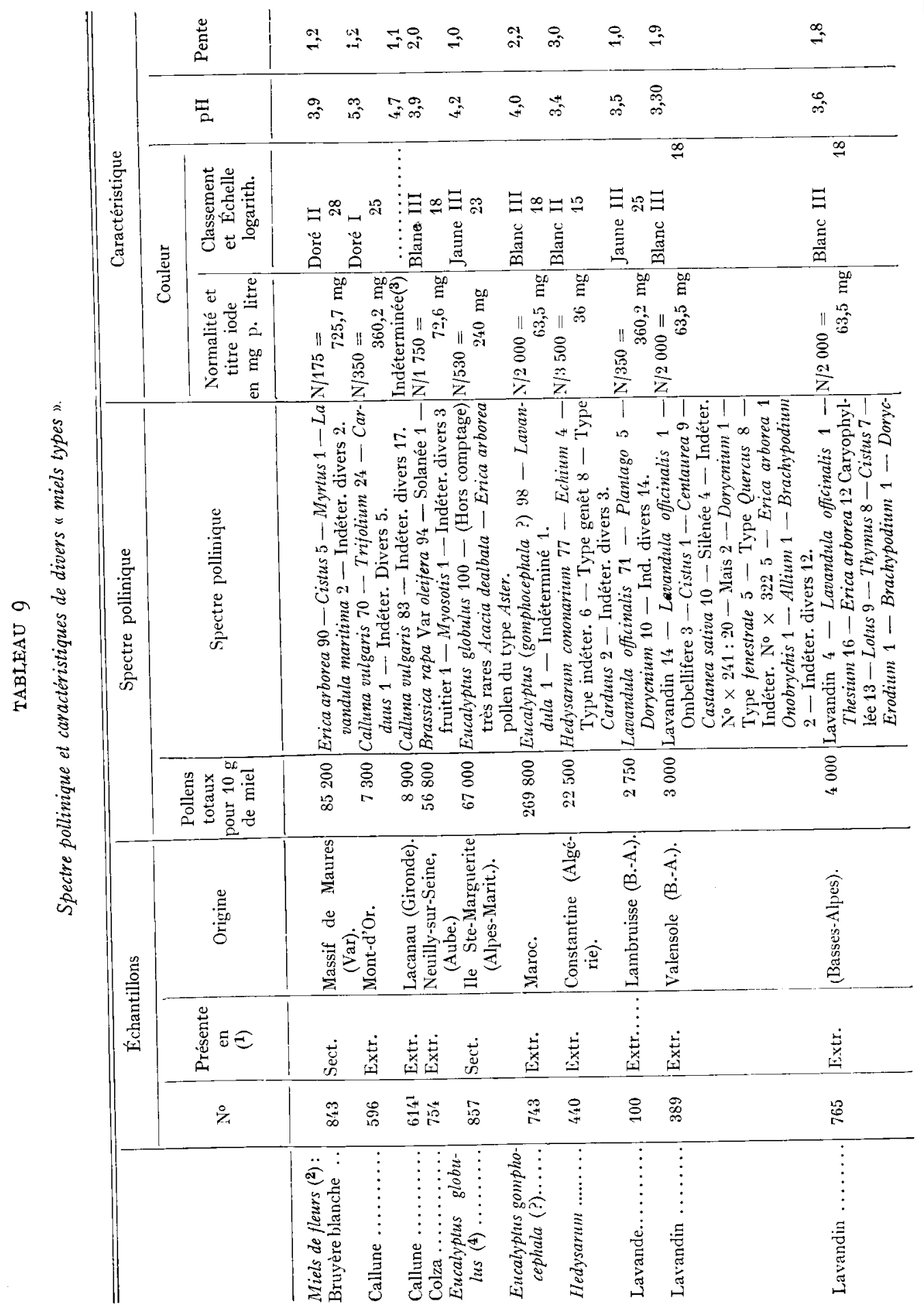




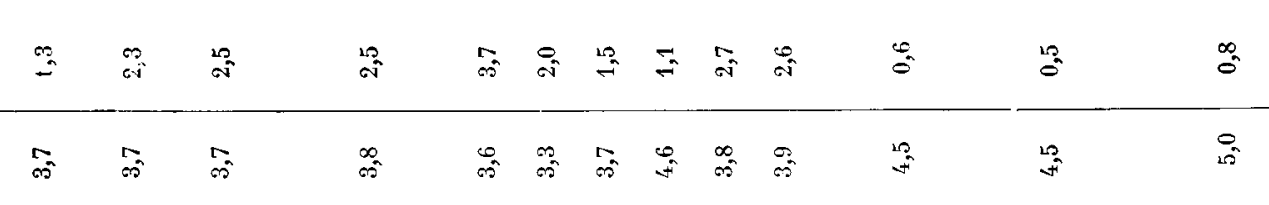

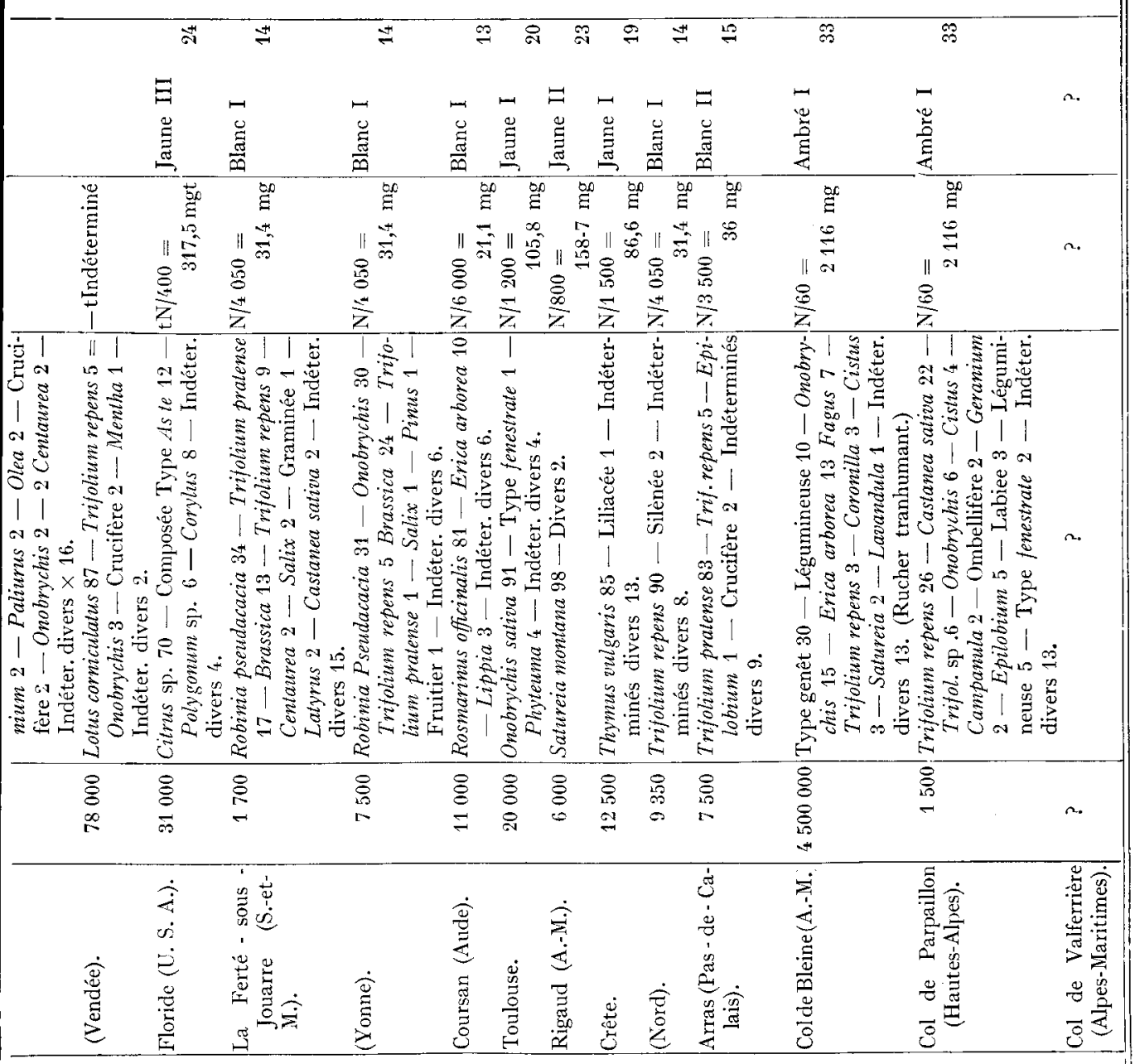

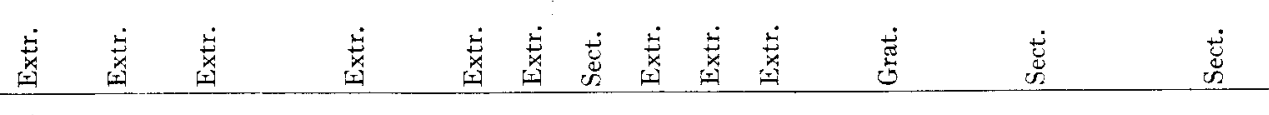

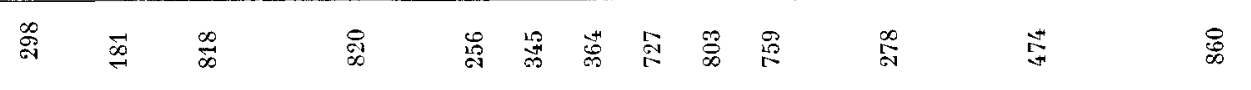

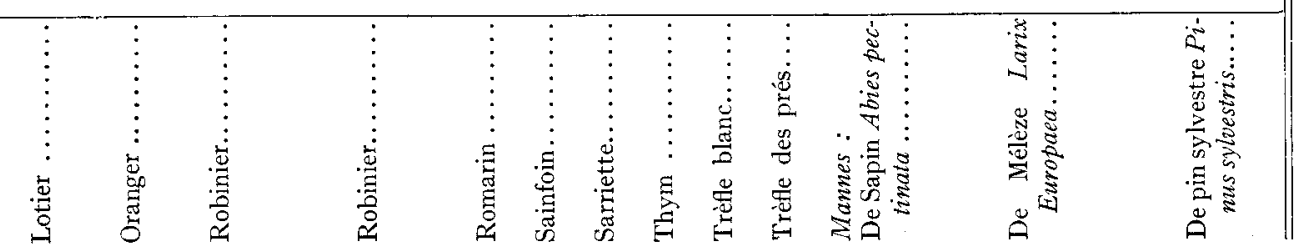




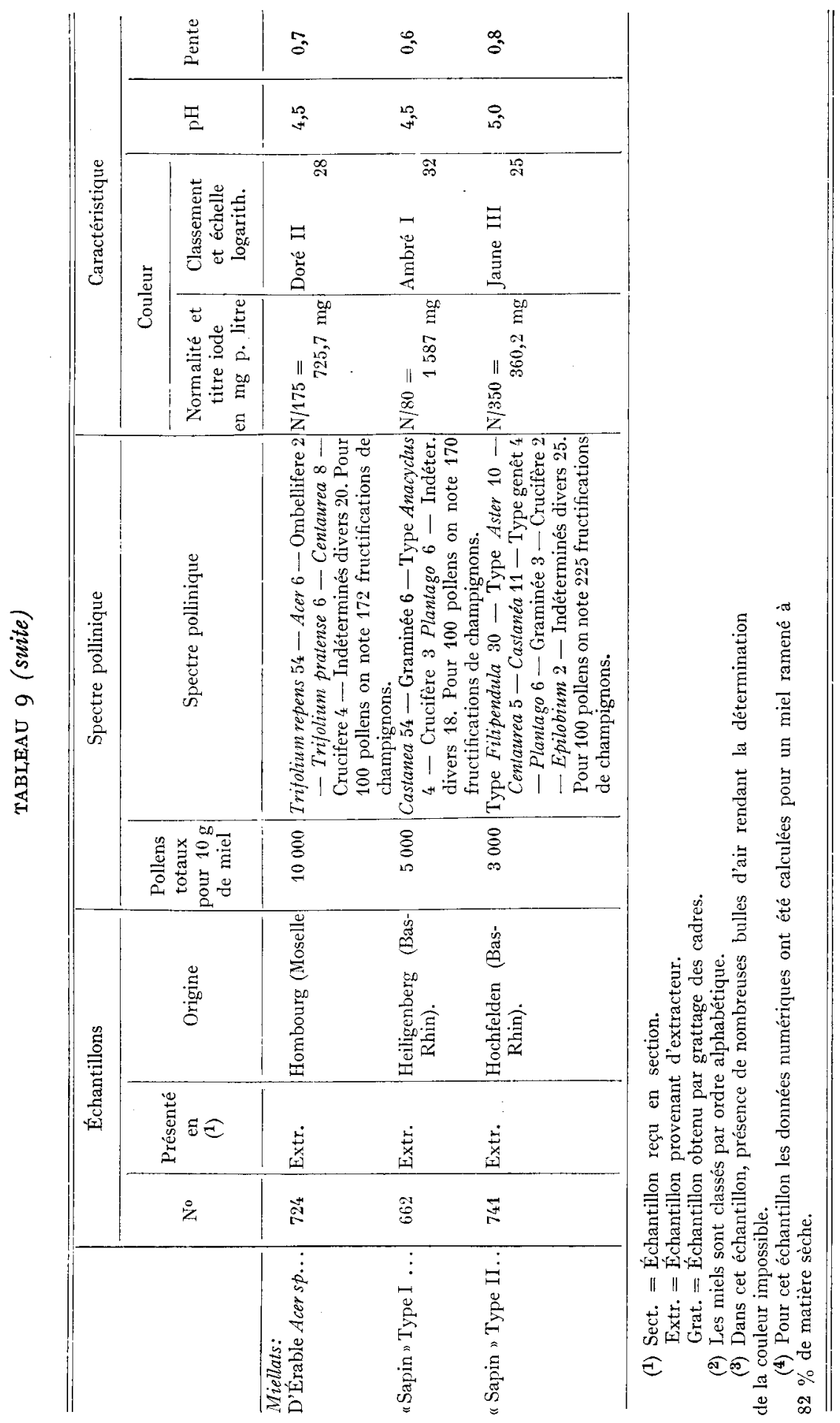


tillons présentant toutes les garanties de récolte et dont les caractéristiques ont été retrouvées sur plusieurs autres.

On trouvera au tableau 9 les caractéristiques de ces miels et miellats. Toutefois il convient de remarquer que la grande majorité des échantillons dont nous disposions étaient des miels d'extracteur qui à cause des mélanges inévitables provoqués par cette opération, correspondaient plus à des miels à dominance qu'à des miels types.

Aussi serait-il souhaitable de poursuivre ces travaux, en utilisant des échantillons prélevés directement dans des cadres, qui auraient été placés dans les ruches au moment d'une miellée.

Avec de tels échantillons les risques de présence de miels différents et surtout de miellats, parfois bien difficile à déceler, seraient moins grands et la détermination des caractéristiques plus sûre.

\section{CONCLUSION.}

I1 ressort de ces observations, que les miels types, provenant de diverses plantes ont des valeurs caractéristiques différentes pour la couleur, la courbe de neutralisation et à un degré moindre le $\mathrm{pH}$ initial. Ces remarques sont valables aussi bien pour les miels de fleurs que pour les miellats et mannes.

Elles confirment aussi les travaux de Maurizio qui a démontré que selon leur origine botanique les miels peuvent être considérés unifloraux à des taux différents de pollen principal dans le spectre pollinique.

En conclusion nous considérons que l'assurance de 1'origine botanique d'un miel repose avant tout sur l'identification des pollens qu'il contient, mais que l'évaluation de sa couleur et de la pente calculée sur la courbe de neutralisation des acides libres, apportent une confirmation nécessaire aux résultats de l'analyse pollinique.

Reçu pour publication en septembre 1960

\section{RÉFÉRENCES BIBLIOGRAPHIQUES}

Barbilir E. C., I954. Observations sur l'analyse pollinique des niels et sur leur origine. Reri. Fr. Apic. 108, 989-99I.

Barbier E. C., Valin J., 1957. Détermination de la couleur des miels. Ann. Fals. Fraud. 58\%-588, 400-4II.

Beutler R., 1953. Vectar. Bee World, 34, 6, 106-116; 7, 128-1 36;8, 156-162.

Cuistov V. C., 1954. Acidité et propriétés tempon des miels (en russe). Pchelovodstvo, 2, $28-32$.

Cocker I., I951-1952. The enzymatic production of acid in honey. j. Sci. Fd. Agric. 9, 4I 1-4I4.

Faegri K., Iversen J., I950. 'Text book of modern pollen analysis. Munsgaard E., Copenhagen.

Louvfaux J., 1958. Recherches sur l'origine dans le miel du pollen des plantes entomophiles dépourvues de nectaires. Ann. Abeille, 2, 89-92.

MaURizio A., r939. Untersusuchungen zur quantitativen pollenanalyse des Honigs. Milt. ans $d$. Geb. Lebensm. Unters. u. Ilyg., 30.

Maurizio A., 1949. Pollenanalytische Untersuchungen an Honig und Pollenhöschen. Beih. Schreeiz. Bienentzg. 2, I8, A. Beiträge zur quantitaven Poollenanalyse des Honigs. 320-42 I.

MaUrizio A., I955. Beitraege zur quantitativen pollenanalyse des Honigs. 2 - Absoluter gehalt pllanzlicher bestandteile in tilia und labiaten Ionigen. A. Bienentorsch. 3, 2, 32-39.

MaURizio A., I958. Beitrage zur quantitativen pollenanalyse des Honigs. 3. Absoluter Gehalt pflanslicher Bestandteile in Fsparsette, Luzerne, Orangen und Rapshonigen, Ann. Abeille, 2, 9.3-106.

Milum G. V., I948. Some factors affecting the colour of Honey. Iorea Rep. State 1piarist for I948-1949, 52-64.

Pangaud Cl. ig6o. Etude sur l'acidité des miels et des miellats. Thèse Clermont-Ferrant (sous presse). 